\title{
Immediate Implantation in the Anterior Maxilla: A Case Report and Discussion of Advantages and Pitfalls
}

\author{
${ }^{1}$ TV Narayan, ${ }^{2}$ Sarita Joshi Narayan \\ ${ }^{1}$ Professor and Head, Department of Oral Pathology and Microbiology, The Oxford Dental College and Hospital, Bomanahalli \\ Bengaluru, Karnataka, India \\ ${ }^{2}$ Professor and Head, Department of Periodontics, Rajiv Gandhi Dental College, Cholanagar, Hebbal, RT Nagar Post, Bengaluru \\ Karnataka, India
}

Correspondence: TV Narayan, Professor and Head, Department of Oral Pathology and Microbiology, The Oxford Dental College and Hospital, Bomanahalli, Bengaluru, Karnataka-560068, India, e-mail: tvnarayan@yahoo.com

\begin{abstract}
Esthetic implant therapy in the anterior maxilla is dependent on multiple factors including the lip drape, the implant position and the available peri-implant soft tissue to name a few. The latter two factors become increasingly important in a patient with a high lip line where a fraction of a millimeter can spell success or disaster. This is further confounded by the fate of the extraction socket. The shift in paradigm from FPDs to implants has placed special emphasis on the management of the extraction wound and timing of implant placement. This report describes a case of immediate implantation into a fresh extraction socket of a maxillary central incisor with immediate provisional Maryland - Rochette bridge, and discusses the advantages and pitfalls of immediate implant placement.
\end{abstract}

Keywords: Maryland bridge, immediate implant placement.

\section{INTRODUCTION}

Planning implant treatment for a hopeless tooth starts prior to extraction. The timing and technique of the extraction to the timing of implant placement all play critical roles in treatment outcome. The timing of implant placement is the fourth dimension in treatment planning for implants after the three dimensions of available bone.

Esthetic implant therapy in the anterior maxilla is dependent on multiple factors including the lip drape, the implant position and the available peri-implant soft tissue to name a few. The latter two factors become increasingly important in a patient with a high lip line where a fraction of a millimeter can spell success or disaster. This is further confounded by the fate of the extraction socket. The shift in paradigm from FPDs to implants has placed special emphasis on the management of the extraction wound and timing of implant placement.

With immediate implants becoming increasingly predictable as the parameters for successful treatment get better defined each day, and with the considerable reduction in treatment time and increased patient compliance, its relevance in the current context of implant dentistry is ever increasing. Here, report a case of immediate implantation in the maxillary central incisor region and discuss the various factors that predicate success.

\section{CASE REPORT}

A 30 years old male patient in good health, reported with pain and mobility of the maxillary right central incisor. There was a history of trauma sustained about 12 years ago and attempted endodontic treatment about 2 years after the trauma. An intraoral radiograph revealed an apical fracture on 11 , and an obviously aborted attempt at endodontic therapy (Fig. 1). The reason for incomplete RCT was calcification of the chamber and canal. There was no periapical lesion or periodontal bone loss appreciable on the X-ray nor any soft tissue edema clinically. The patient's periotype was assessed and found to be a thick gingival type, with a high scallop and bone sounding for the labial plate revealed the crest of labial bone $3 \mathrm{~mm}$ apical to the gingival margin. The mobility was attributed to the fracture and extraction followed by an attempt at immediate implantation was planned after obtaining the appropriate consent. The patient had an edge to edge occlusion with no incisal guidance what soever and impressions were recorded for fabrication of a provisional Maryland-Rochette bridge with an ovate pontic in Adoro (Ivoclar-Vivadent) without any tooth preparation, after scoring the model down to about $3 \mathrm{~mm}$ apical to the crest of the gingiva. The patient was scheduled for surgery and prescribed Amoxycillin $500 \mathrm{mg}$ tid 1 day preoperatively. The extraction was performed under 


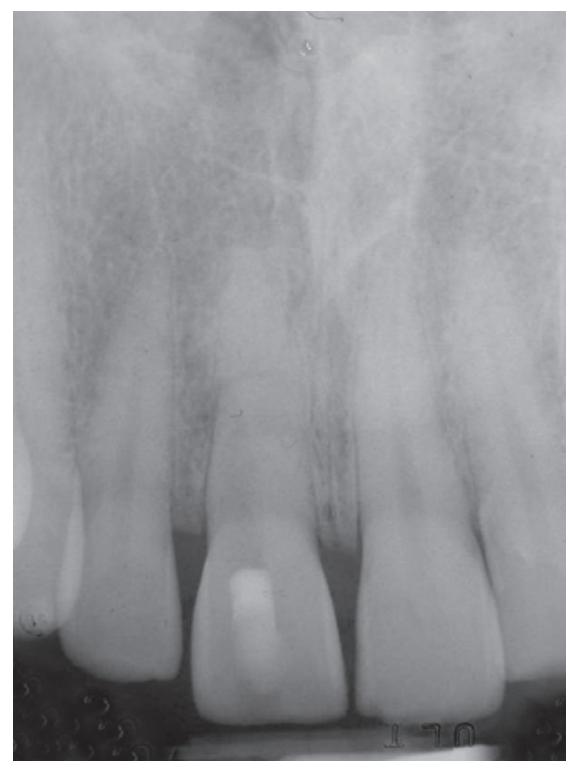

Fig. 1: Preoperative radiograph

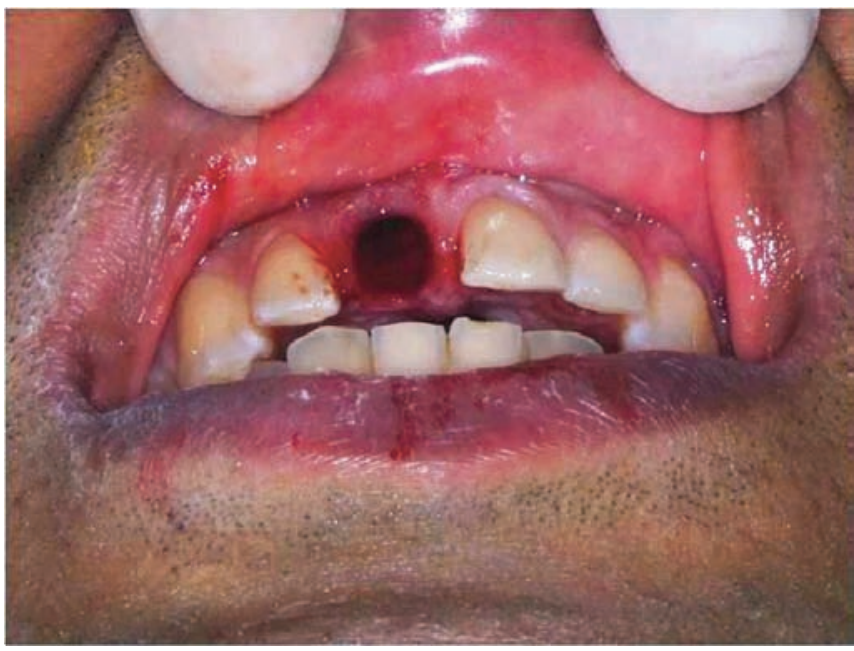

Fig. 2: Extraction socke

local anesthesia using periotomes and luxators with appropriate precautions to ensure that the labial plate of bone was not traumatized. The extraction socket was carefully examined for dehiscences and fenestrations and debrided of residual periodontal fibers using curettes (Fig. 2).

A self tapping tapered implant of $5 \mathrm{~mm}$ diameter and $13 \mathrm{~mm}$ length (EZ-Hi-Tec, Israel) was placed after preparing an osteotomy along the palatal wall of the socket and $3 \mathrm{~mm}$ beyond the apex of the socket to ensure a palatal orientation of the implant with no contact between the implant and the labial bone plate (Figs 3 and 4). The insertion torque achieved was between 35 and $40 \mathrm{Ncm}$ which is adequate for immediate loading. However, there was a gap of about $2 \mathrm{~mm}$ between the implant crest and the labial plate so it was

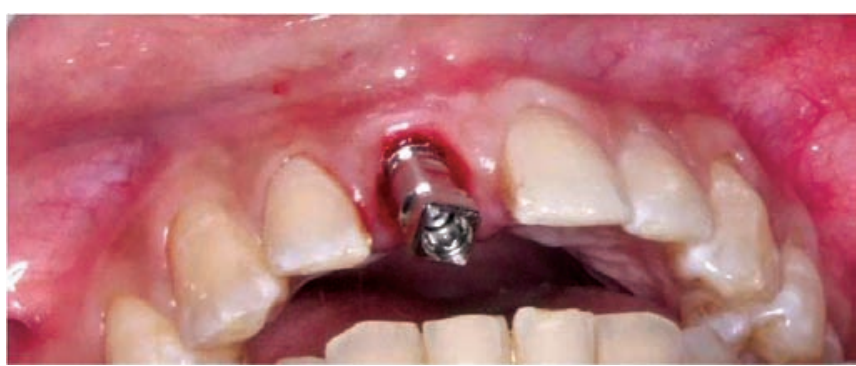

Fig. 3: Immediate implant placed

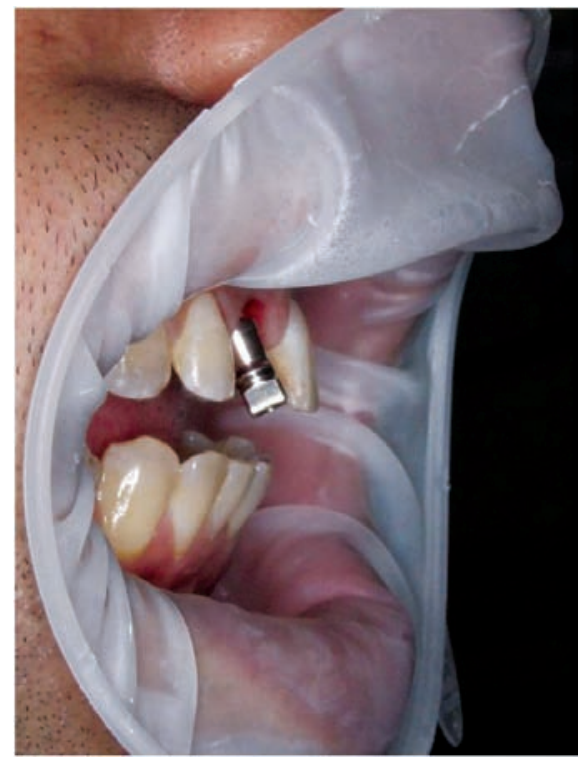

Fig. 4: Profile view of implant to show the palatal orientation

decided to use the Bio-Col technique ${ }^{1}$ and use the prefabricated provisional bridge. The gap was filled with BioOss (Geistlich, Switzerland) and Collaplug (Zimmer Dental, USA) was placed over the cover screw and compressed.The provisional bridge was bonded to the unprepared enamel after acid etching, using Unicem (3M ESPE, USA). No sutures were used and the collaplug was held in place solely by the ovate pontic (Figs 5 and 6 ).

The immediate postoperative period was uneventful and the patient returned after 4 months for the definitive implant restoration. The soft tissue around the pontic was healthy, with no signs of inflammation nor recession. The radiograph at this stage revealed nothing untoward. The provisional bridge was retrieved and a thin layer of soft tissue was seen over the coverscrew of the implant (Fig. 7) which was curetted away, and a closed tray technique was used after connecting the impression post to record the impression in Impregum Penta (3M ESPE). The pontic was relined with composite to contact the cover screw and bonded back. A Platform switch was performed using a standard abutment of the $3.5 \mathrm{~mm}$ platform and the final restoration in PFM 


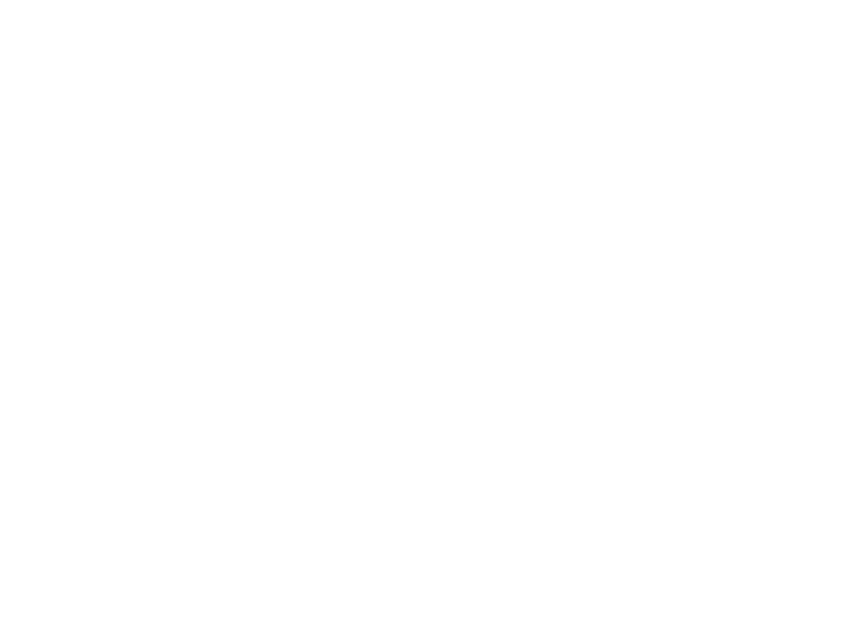

Fig. 5: Provisional bonded bridge with ovate pontic

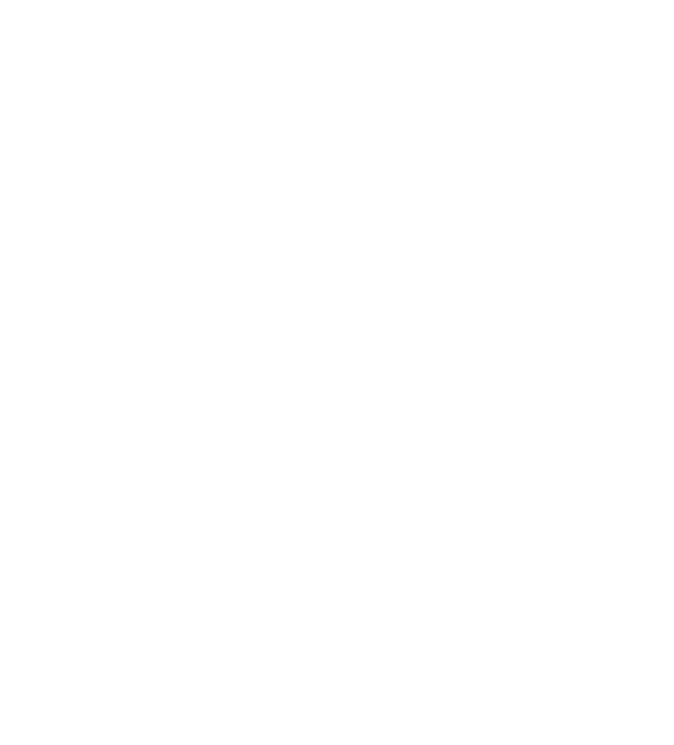

Fig. 6: Radiograph showing bonded bridge and implant

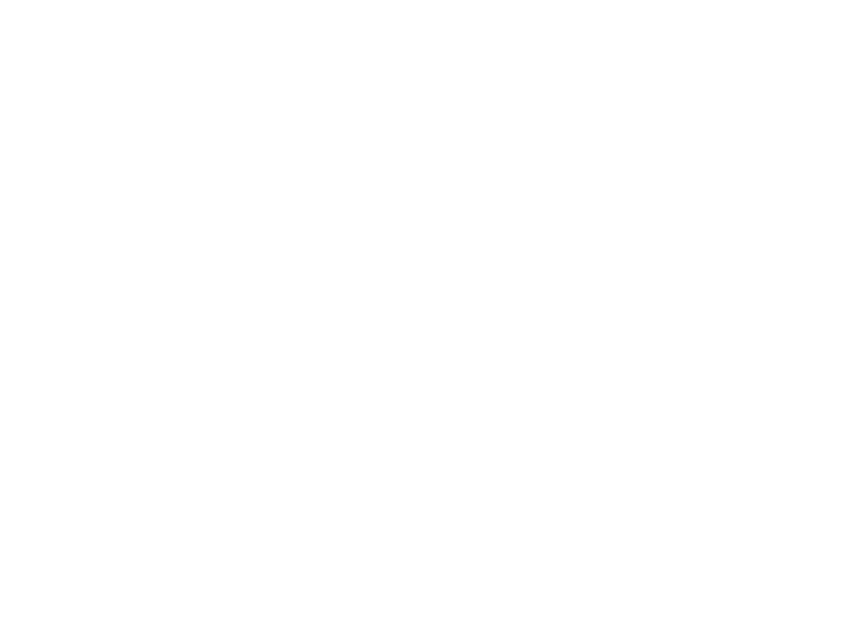

Fig. 7: After removal of provisional bridge and exposure of cover screw was cemented a week later (Fig. 8). A Periapical X-ray at this stage showed that the restoration was short of the prepared margin which was unacceptable considering current standards of practice. However, due to paucity of time, since the patient was travelling the same day, it was decided that the crown would be changed at his next visit. At 1 year follow-up, after functional restoration, the periimplant soft tissue was healthy, with no signs of inflammation and the zenith was at a lower level than of the neighboring incisor and no bone loss radiographically and again owing to the patient's schedule the restoration could not be repeated (Figs 9 and 10). At the time of writing this article, the implant has been in function for 2 years.

\section{DISCUSSION}

Ever since Lazzara ${ }^{2}$ reported on the surgical advantages of immediate implant placement, it has become an increasingly popular treatment modality particularly with teeth of poor prognosis in an otherwise healthy setting of the anterior maxilla. The potential benefits include maintaining the integrity of the labial plate of bone and if adequately temporized, maintaining the volume and position of the soft tissues. ${ }^{3}$ A number of articles in literature expound the purported virtues and demerits of immediate implantation. ${ }^{4-6}$

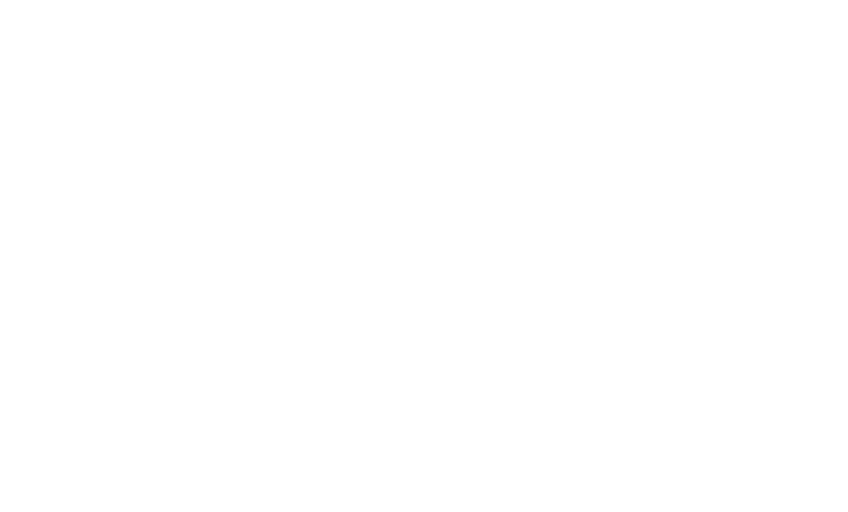

Fig. 8: PFM crown cemented

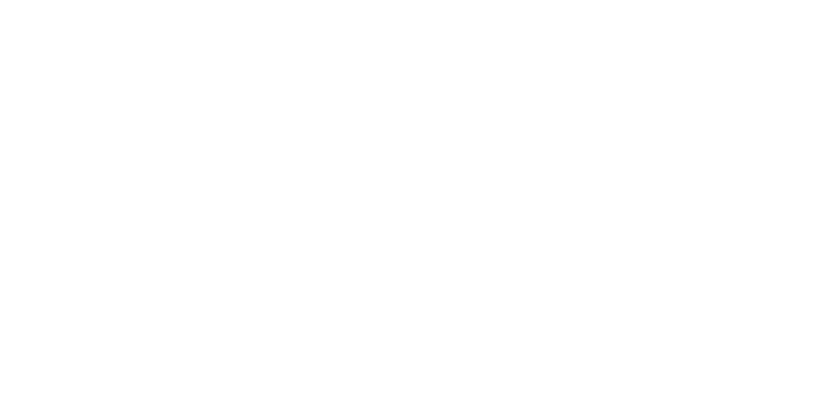

Fig. 9: One year recall photograph 


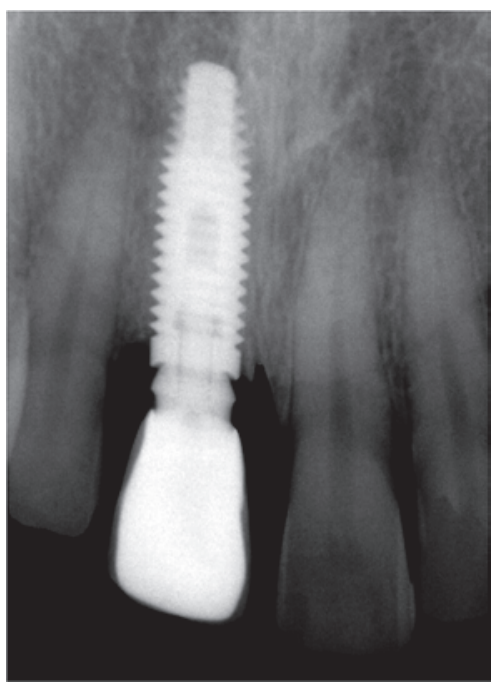

Fig. 10: One year recall radiograph

In the 2008 Cochrane review on immediate implants, Esposito and his coworkers observed that immediate and immediate delayed implants may offer some advantages over conventional delayed implants in terms of patient satisfaction and esthetic outcome possibly by preserving alveolar bone. ${ }^{7}$

Discussing the protocol for immediate implant placement into extraction sockets, Schwartz and Chausa ${ }^{8}$ concluded that:

1. Immediate implants have a high-rate of survival, ranging from 93.9 to $100 \%$.

2. Implants must be placed 3 to $5 \mathrm{~mm}$ beyond the apex to achieve maximum stability.

3. Implants must be placed as close to the alveolar crest as possible (0 to $3 \mathrm{~mm}$ ).

4. There is no consensus regarding gap filling or the best grafting material.

5. The use of a membrane does not imply better results; on the contrary, membrane exposure may cause complications.

6. The absolute need for primary closure is to be established.

To add to these observations, a few additional guidelines would be:

A thorough examination of the periotype prior to:

1. Atraumatic extraction.

2. The presence of an intact labial plate.

3. Avoid raising a flap as far as possible.

4. Orient the osteotomy palatally.

5. Use an appropriate size of implant to ensure engagement of bone 3 to $5 \mathrm{~mm}$ beyond the apex and no contact/ pressure on the labial plate; The aim is not to try and fill the entier socket with the implant.
In the preceding case report, all conditions were favorable and the guidelines could be followed to the letter. The use of a graft material for gap filling was a matter of personal preference, rather than a specific indication. The Bio-Col protocol was used based on its sound biologic rationale.

The choice of temporary restoration was owing to the advantage offered by the clinical situation of no incisal guidance and considering that bone - implant contact in immediate implant is considerably less than in conventional delayed implants, the chosen temporization ensured that no micromovement was possible at the bone implant interface.

The thick gingival type in this case ensured that there was no recession of soft tissue even 1 year after extraction, with the platform switch possibly furthering this cause.

\section{CONCLUSION}

Immediate implants are increasingly predictable and as illustrated in this case, with all parameters being favorable to success, can provide esthetically superior results at least in the short-term follow-up period. More Randomized control trials on large numbers of implants will be needed to establish the long-term esthetic predictability of this treatment modality.

\section{REFERENCES}

1. Sclar Anthony G, Soft tissue and esthetic considerations in implant therapy: Quintessence 93-103.

2. Lazzara RJ, Immediate implant placement into extraction sites: Surgical and Restorative advantages. Int J Periodont Rest Dent 1989;9(5):332-43.

3. Garber DA, Salama, Salama MA. Two Stage versus one stage: Is there really a controversy? J Periodontol 2001;72:417-21.

4. Saadoun Andre P, Sebbag Phillipe Compendium 2004;25(4): 277-96.

5. Mazor Ziv, Peleg Michael, Redlich Meir JADA 1999;130: 1767-70.

6. Grunder U, Polizzi G, Gaene R, et al. A 3 years prospective multicenter follow-up report on immediate and delayed immediate placement of implants. Int J Oral and Maxillofac. Implants 1999;14:210-16.

7. Esposito MAB, Koukoulopoulou A, Coulthard P, Worthington HV Interventions for replacing missing teeth: Dental implants in fresh extraction sockets (immediate, immediate-delayed and delayed implants) Cochrane Database of Systematic Reviews 2006, Issue 4. Art. No.: CD005968. DOI: 10.1002/ 14651858.CD005968.pub2.

8. Schwartz-Arad D, Chausa G. The ways and wherefores of immediate placement of implants into fresh extraction sockets: A literature review. J Periodontol 1997;68(10):915-23. 